\title{
On the Performance of TETRA DMO Short Data Service in Railway VANETs
}

\author{
Andreas Lehner • Cristina Rico García • \\ Thomas Strang
}

C) Springer Science+Business Media, LLC. 2012

\begin{abstract}
In this paper we present the results of an empirical study investigating the performance of TETRA (TErrestrial Trunked RAdio) in a Vehicular Ad-hoc Network (VANET) for safety related railway traffic applications. The Short Data Service (SDS) of TETRA in the Direct Mode Operation (DMO) allows an infrastructure-less exchange of traffic relevant information between vehicles in range of the communication. The propagation channel in case of such a direct (base station free) communication between railway vehicles underlies specific effects, which are not equivalent to other well known terrestrial mobile scenarios. We will present measurements covering urban, suburban and rural environments along a regional railway network in the south of Bavaria. Beside different operational conditions such as front, rear, and flank approaches of trains, we investigated several topological scenarios on both, single and double track sections along the line. We will also discuss the observed characteristic changes in narrow band signal attenuation and Doppler spectra for passages through forests, hilly areas, stations and a tunnel. We determined statistics for the transmission delay of messages and the message erasure rates for single and multi user access on a single common carrier for different transmission intervals. Finally, the quality of service for the envisaged safety applications is assessed.
\end{abstract}

Keywords TETRA - DMO - Short data service - VANET - Propagation - Channel · Measurement · UHF · Train-to-train · Railway · Path loss · Model · V2V · MAC · RCAS

\footnotetext{
A. Lehner $(\varangle) \cdot$ C. Rico García · T. Strang

German Aerospace Center DLR, Institute for Communications and Navigation, Muenchner Str. 20, 82234 Wessling, Germany

e-mail: andreas.lehner@dlr.de

C. Rico García

e-mail: cristina.ricogarcia@dlr.de

T. Strang

e-mail: thomas.strang@dlr.de
} 


\section{Introduction}

Vehicular Ad-hoc NETworks (VANETs) enable a range of novel safety related applications in transportation based on direct communication among vehicles. As an example the German Aerospace Center (DLR) is currently developing a Railway Collision Avoidance System (RCAS) [1,2], which will allow a train driver to have an up-to-date accurate knowledge of the traffic situation in the vicinity, and act in consequence. The basic idea of RCAS is to calculate the own position and movement vector and broadcast this information as well as additional data such as vehicle dimensions to all other trains in the area. The train driver's cabin is equipped with a display showing the position of the other vehicles in the region. Computer analysis of the received information together with the own status allows to detect collision threats, displaying an alert signal and advising the driver how to avoid the danger. In order to work reliable, the system requires a communication range that is in all cases larger than twice the maximum braking distance [3], which is below $1.5 \mathrm{~km}$ in regional networks.

Another challenge in the design of VANETs is to effectively control the common media access. In cellular networks the media access is usually controlled by base stations in a centralized manner. Cell arrangement and frequency reuse is optimized to cause minimum interference from nodes in neighboring cells. The character of VANETs requires distributed solutions that strongly depend on the node density and on the communication range, respectively the interference range of nodes in the neighborhood [4]. Thus, it is important to have accurate knowledge of path loss and fading characteristics under realistic conditions, e.g. when regional lines meet shunting areas or large stations with high user densities, where both, communication with a fast approaching train and the information exchange to the multiple nodes in the close vicinity have to be reliably maintained at the same time.

Although it does not scale for areas with dense railroad traffic, the first RCAS system demonstrator was built using TETRA (TErrestrial Trunked RAdio) mobile terminals broadcasting on a single channel. Since the provided data rate by the TETRA standard is very similar to the RCAS system requirements [5], this allowed us to investigate the message transmission performance under realistic propagation conditions. In this way both, the performance evaluation of the TETRA DMO-SDS messaging for train-to-train conditions and the corresponding channel characterization can be achieved.

TETRA is a European Telecommunications Standard Institute (ETSI) standard that was specifically designed for emergency services, public safety and for military usage, to provide robust and secured communication even under disaster conditions. Based on a $\pi / 4$ DQPSK (Differential Quadrature Phase Shift Keying) digital modulation scheme, TETRA has a high spectral efficiency comparable to the one of GSM (Global System for Mobile communications), but is limited to lower data rates due to a $25 \mathrm{kHz}$ channel spacing $(200 \mathrm{kHz}$ in GSM). TETRA operates in the UHF frequency band. Although primarily designed for voice services, the TETRA system supports several types of data communication, including message based communication such as status messages and Short Data Services (SDS) [6]. Particularly interesting for infrastructure-less data exchange between vehicles in terrestrial transport, TETRA Mobile Stations (MS) can directly communicate with each other in the Direct Mode Operation (DMO) offering both, point-to-point and point-to-multipoint transmissions. Another advantage for safety critical communication is the ability of a very fast call setup. Radio parameters and several mechanisms in the protocols shall ensure successful communication during overload situation. The reserved frequency bands in the lower UHF band permits much larger communication ranges compared to GSM or UMTS. In the following we summarize the features that make TETRA beneficial for the envisaged type of applications, and initiated the study we are presenting in this paper: 
- High spectral efficiency: $25 \mathrm{kHz}$ channel bandwidth with net data rates of up to $28.8 \mathrm{k} \mathrm{bit} / \mathrm{s}$ [6], fulfilling the requirements on the system data rate of safety related railroad traffic applications [6].

- Works at high relative speeds $>400 \mathrm{~km} / \mathrm{h}$.

- Mobile station to mobile station communication is implemented in the DMO. Both, point-to-point and point-to-multipoint transmissions are possible.

- Supports data communication, like status messages and SDS.

- Low UHF frequencies permit a communication range of several kilometers.

- Very fast call set-up-typical less than $250 \mathrm{~ms}$ for a single node call.

- The system contains several mechanisms, designed into the protocols and radio parameters, to ensure communication success even during overload situations. The system also supports a range of emergency calling modes.

Although TETRA was primarily designed for communication of mobiles via base stations, the DMO recently led to a growing number of applications. However, the special propagation and interference conditions that arise in DMO are not particularly reflected in the system design, thus no realistic assessment of the performance can be derived from the measures and simulations provided in the TETRA standard documents. On the other hand, the design of Vehicle-to-Vehicle (V2V) communication systems requires accurate knowledge about the specific propagation characteristics. These characteristics are certainly different to the widely measured and accurately modeled conditions in cellular terrestrial networks, where antennas of base stations are at advantageous heights above ground. In V2V communication, antennas are usually placed on top of the vehicles, which is significantly lower than a cellular base station antenna. But not only has the low height of antennas raised uncertainties. Especially in the railway environment we face very specific conditions that influence the wave propagation along the lines. Shadowing by platforms with roofs in stations, or reflections from other railway vehicles might cause high losses. On the other hand, cuttings along the lines of railway networks with relatively large curve radii, catenaries, and tunnels are likely to have guidance effects [7] that could result in unexpected low path loss exponents at larger distances.

In TETRA DMO the media access has not been particularly designed for multi-broadcast communication as it is the concept in RCAS, where each mobile periodically transmits traffic information to the other mobiles. Nevertheless, stressing parameterization conform to the standard can achieve reasonable performance as we will show in this study. Moreover, since TETRA uses a TDMA (Time Division Multiple Access) scheme, novel Media Access Control (MAC) protocols [8] are applicable in principle, which can offer further improvements in Quality of Service (QoS).

The remainder of this paper is organized as follows. Sections 2 and 3 describe TETRA DMO and SDS principles. In Sect. 4 we introduce our TETRA evaluation platform. The railway measurement campaign and the obtained propagation channel characteristics are presented in Sects. 5 and 6. In Sect. 7 we analyze the performance of TETRA in the outlined scenario. Finally, Sect. 8 concludes the paper and highlights the results.

\section{TETRA DMO: Direct Mode Operation}

In Direct Mode Operation (DMO) mobile stations (MS) communicate with each other by using their radio interface for direct communication (MS to MS) - either simple point-topoint or point-to-multipoint. In this mode intercommunication of MSs is independent of any 
mediation by a base station network [9]. A major criterion in the development of TETRA DMO was to provide a simple but robust system using parameters as close to the TETRA TMO (Trunked Mode Operation) as possible.

A TETRA DMO channel is the Direct Mode radiofrequency carrier where any DMO operation takes place. There are three states for a Direct Mode channel [10]:

- Free: when there is no call or message being sent. Thus, any MS may use it.

- Occupied: when there is a call in progress.

- Reserved: when there is presence of a channel reservation signal.

In absence of any base station there are two roles in TETRA DMO in order to achieve the correct synchronization between MSs: The master and the slave. The master will be the MS which initiates the communication. And the slave or slaves are the other stations that will be listening to the DMO Channel [11].

As the master, this MS provides the synchronization reference to all the other stations involved in this call. At the beginning of a call, during a call transaction or during channel reservation, the master will transmit synchronization bursts to enable any receiving MS on the same channel to synchronize itself in terms of frequency and time. In every situation the slave MS shall align its transmission to the timeslots and carrier frequency dictated by the transmission of the master MS.

A burst is a small amount of time on a radiofrequency carrier that is modulated by a data stream. There are tree types of bursts in TETRA DMO: DMO Linearization burst (DLB), DMO Normal Burst (DNB) and DMO Synchronization Burst (DSB). The DLB has no useful bits and is used to linearize the transmitters of the mobile stations. The DNB has two blocks of 216 bits for carrying data, together 432 bits. The rest of the burst fields are for phase adjustment and training sequences. The DSB is used to synchronize the mobile stations participating in the communication. It also allows transportation of two blocks of data, one of 120 bits and the other of 216 bits, in total 336 bits. For further details we refer to [12].

\section{SDS: Short Data Service}

The TDMA scheme in TETRA provides four physical channels per carrier, and carriers are separated by $25 \mathrm{kHz}$. The basic radio resource is a time slot lasting $14.167 \mathrm{~ms}(85 / 6 \mathrm{~ms})$ transmitting information at a modulation rate of $36 \mathrm{kbit} / \mathrm{s}$. The time slot duration, including guard and ramping times equals the duration of 255 symbols ( $510 \mathrm{bits})$. The physical content of a time slot is carried by a burst (DLB, DNB or DSB). One frame consists of four slots and has a duration of $170 / 3 \approx 56.67 \mathrm{~ms}$. Additionally, as depicted in Fig. 1, a multiframe structure of 18 frames (lasting 1.02s) is defined that allows the introduction of associated control channels together with their corresponding traffic channels for speech and data [13].

Three types of physical channels are defined:

- Control Physical channel (CP) is a physical channel carrying exclusively control channels. Two types of $\mathrm{CP}$ are defined: Main Control $\mathrm{CHannel}(\mathrm{MCCH})$, which is located on the timeslot 1 of the main carrier, and Secondary Control CHannel ( $\mathrm{SCCH})$, which may be used to extend the signaling capacity of the $\mathrm{MCCH}$ and may only be assigned when the MCCH is used.

- Traffic Physical channel (TP) is a physical channel carrying Traffic Channel (TCH).

- Unallocated Physical channel (UP) is a physical channel not allocated to one or more MS—e.g. used for broadcast. 


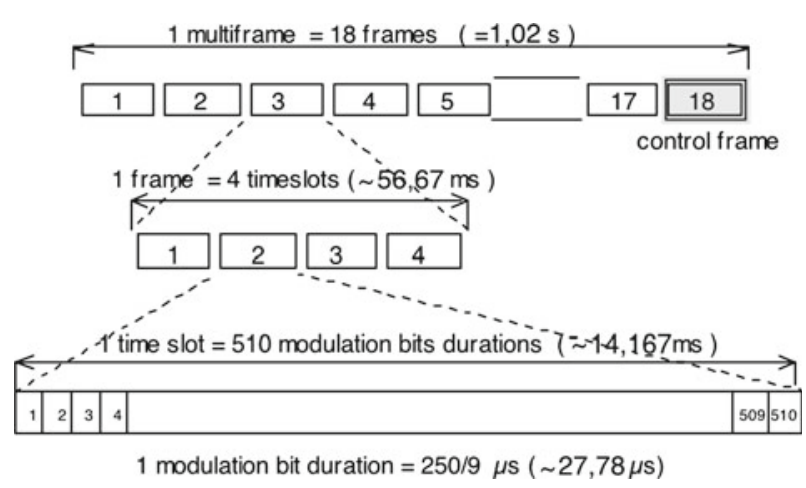

Fig. 1 TETRA multiframe structure [13]

\begin{tabular}{|c|c|c|c|c|c|c|c|c|c|c|c|c|c|c|c|c|c|c|c|c|c|c|c|c|}
\hline \multirow{3}{*}{$\begin{array}{l}\text { Frame \# } \\
\text { Slot \# } \\
\text { Channel }\end{array}$} & \multicolumn{4}{|c|}{17} & \multicolumn{4}{|c|}{18} & \multicolumn{4}{|c|}{1} & \multicolumn{4}{|c|}{2} & \multicolumn{4}{|c|}{3} & \multicolumn{4}{|c|}{4} \\
\hline & 1 & 2 & 3 & 4 & 1 & 2 & 3 & 4 & 1 & 2 & 3 & 4 & 1 & 2 & 3 & 4 & 1 & 2 & 3 & 4 & 1 & 2 & 3 & 4 \\
\hline & sdu & sdu & sdu & sdu & sdu & sdu & sdu & sdu & $\mathrm{sd}$ & & & & $\mathrm{sd}$ & & $p ?$ & & $\mathrm{sd}$ & & & & sdu & sdu & sdu & sdu \\
\hline \multirow{3}{*}{$\begin{array}{l}\text { Frame \# } \\
\text { Slot \# } \\
\text { Channel }\end{array}$} & \multicolumn{4}{|c|}{5} & \multicolumn{4}{|c|}{6} & \multicolumn{4}{|c|}{7} & \multicolumn{4}{|c|}{8} & \multicolumn{4}{|c|}{9} & \multicolumn{4}{|c|}{10} \\
\hline & 1 & 2 & 3 & 4 & 1 & 2 & 3 & 4 & 1 & 2 & 3 & 4 & 1 & 2 & 3 & 4 & 1 & 2 & 3 & 4 & 1 & 2 & 3 & 4 \\
\hline & sdu & sdu & sdu & sdu & $\mathrm{sd}$ & & & & sd & & & & sd & & $\mathrm{p}$ ? & & & & & & & & & \\
\hline
\end{tabular}

Fig. 2 Call sequence for set-up of a SDS call for unacknowledged data [10]

Status messages and SDS are provided over the system's Main Control CHannel (MCCH). Status message service provides pre-defined messages from a limited list, while the userdefined SDS allows transmitting messages up to 256 bytes of binary arbitrary data. There are four types of SDS, the types 1, 2 and 3, have a fixed size of 16, 32 and 64 bits respectively. The SDS type-4 is the one service with variable size up to 2,047 bits.

\subsection{TETRA DMO SDS Call Setup}

The SDS service can be used either with an unacknowledged or acknowledged protocol [10], but for the communication of data from point to multipoint, the only available protocol is the unacknowledged one. A MS wishing to send an unacknowledged short data message follows the procedures to ascertain the state of the channel. Provided that the channel is found to be in the state "free", the MS establishes the channel synchronization and simultaneously its role as "master" by transmitting a sequence of DM-SDS UDATA (Direct Mode SDS User DATA) message headers using the DSB structure ("sdu" in Fig. 2, with 8 "sdu" being sent in this example). The DM-SDS UDATA message headers contain frame count information which, in the example, defines their position in the timing structure in frames 17 and 18 of the 18-frame cyclic multiframe structure. The master MS then transmits the remaining parts of the short data message ("sd" in Fig. 2), without repetition and using the DNB structure, in slot 1 of the following frames. In this example the remaining parts of the message occupy three slots and are sent in frames 1-3.

For reliability, the master MS may repeat the complete message transmission immediately (without re-checking that the channel is free), and starting again with DSBs. In this example there is one complete message repetition, with the DSBs sent in frames 4 and 5, and the three DNBs sent in frames 6-8. 
Table 1 RFSA characteristics

\begin{tabular}{ll}
\hline Frequency range & $9 \mathrm{kHz}-2.7 \mathrm{GHz}$ \\
Sensitivity & $-130 \mathrm{dBm}$ \\
Dynamic range & $80 \mathrm{~dB}$ \\
IF & $5-25 \mathrm{MHz}$ \\
ADC & $14 \mathrm{bit}, 100 \mathrm{MS} / \mathrm{s}$, IF sampling \\
\hline
\end{tabular}

When a MS sends a DM-SDS UDATA, it becomes master of the channel. If another MS wishes to take the channel to send short data, it must first either wait for the possibility of a changeover or send a pre-emption request ("p?" in Fig. 2) and be granted permission by the current master as described in detail in [10].

\section{TETRA Evaluation Platform for VANETs}

For the measurements and tests described in the following chapters, we developed a software module that accesses TETRA radios through the Peripheral Equipment Interface (PEI) for Quality of Service (QoS) evaluation purposes. The software allows configuration of SDS transmission parameters such as SDS type, priority or the message format, through AT-commands specified in the respective ETSI standard [14]. All transmitted and received data on the PEI can be logged for offline analysis. The software can be used in a distributed manner, allowing to record data on various independent moving test vehicles. The internet Network Time Protocol (NTP) [15] is used to provide synchronization in the order of $10 \mathrm{~ms}$ for the distributed case. Besides recording time stamps of events on the PEI, additional information like position and velocity from sensors such as GPS receivers or Inertial Measurement Units (IMU) can be recorded. Moreover we incorporated an interface to a Radio Frequency Signal Analyzer (RFSA) to gain time and signal strength information from the physical layer. The RFSA (see details in Table 1), which is as well synchronized by NTP, samples the complex signal on the RF carrier and allows the determination of events on the air interface, like e.g. begin and end times of bursts transmitted by the various TETRA radios. In this way transmission conflicts can be identified that are leading to longer transmission delays or message losses.

\subsection{TETRA DMO Radio Configuration Settings}

In DMO a significant amount of channel occupation time is needed in order to synchronize the MSs. The QoS in case of SDS can be improved by increasing the number of transmitted DSBs and by repeating the transmission of the actual data at the cost of reducing the maximum throughput. While typical settings for emergency and public services target the highly reliable and acknowledged delivery of single SDS messages to a group of users with moderate constraints on transmission delay, a much higher throughput has to be achieved to allow multi-broadcast data exchange on a single channel as planned in e.g. RCAS. Although it is essential that frequent updates are received from other vehicles, a message re-transmission is not adequate for our applications, since traffic information is continuously changing-it simply does not make sense to re-transmit data which is outdated in the meantime and an up-to-date information e.g. about the position of a vehicle is available.

Therefore TETRA radio devices in our tests were set to a configuration appropriate for VANETs, where frequent status updates are required. First of all we minimized the number of 
Table 2 Comparison of DMO-SDS parameter settings: VANET versus default

\begin{tabular}{llll}
\hline Type & Parameter & VANET setting & Default/allowed range \\
\hline DM event timer & $\begin{array}{c}\text { DN314 Number of } \\
\text { transmissions of DM SDS }\end{array}$ & 1 & $4 / 1-6$ \\
$\begin{array}{c}\text { UDATA } \\
\text { DM SDS UDATA } \\
\text { FM Maximum Number of }\end{array}$ & 2 & $4 / 2-4$ \\
\hline
\end{tabular}

synchronization bursts to the minimum setting as defined by the TETRA standard, tolerating an increased rate of synchronization errors. In addition we disabled any data retransmission, which furthermore increases the overall failure rate of a single SDS transmission. On the other hand these settings are allowing for shorter update intervals in transmitting new data together with the possibility for multiple other users to broadcast their data in times when the channel is free. In Table 2 we compare our VANET settings to the default settings suggested by the standard.

The TETRA equipment used to achieve the results presented in this study complies with the ETSI TETRA standard and the manufacturer is a member of the TETRA MoU association [16]. Hence the following results are expected to be applicable for other TETRA networks complying with the standard.

\section{Railway Measurement Campaign}

In the design phase of RCAS several questions arose concerning possible hindrances in fulfilling the requirements on the direct train-to-train communication link: Can the communication range be guaranteed to be significantly larger than the braking distances under all topological and operational conditions in different environments? How do passages through urban areas, forests, hilly areas or tunnels affect the statistics of successfully transmitted packets?

In order to be able to get answers to these questions we performed a measurement campaign in an area covering all the interesting environmental, topological and operational aspects. We identified the regional network of the "Bayerische Oberlandbahn (BOB)" to be ideally suited for our measurement campaign and appreciated the extraordinary level of support that we received from $\mathrm{BOB}$ upon our request for cooperation.

\subsection{Railway Network and Environment}

The BOB operates 21 diesel-hydraulic train-sets (17 "Integral" and 4 "Talent"), which carry about 15,000 people per day, with speeds up to $160 \mathrm{~km} / \mathrm{h}$ [17]. The total network has 27 stations and covers $120 \mathrm{~km}$, connecting the metropolis of Munich with the pre-alpine region in the south of Bavaria. Starting from the pure urban environment near the Munich central station the line passes through a tunnel, gradually leaving the city through suburban areas into the forest rich part around Holzkirchen. This part of the network has two tracks, is electrified and is used together with the Munich S-Bahn service. Finally from Holzkirchen to the south three separate lines, which are single track and are not electrified enter the hilly alpine upland area. Figure 3 shows two typical environmental conditions on the BOB network. 

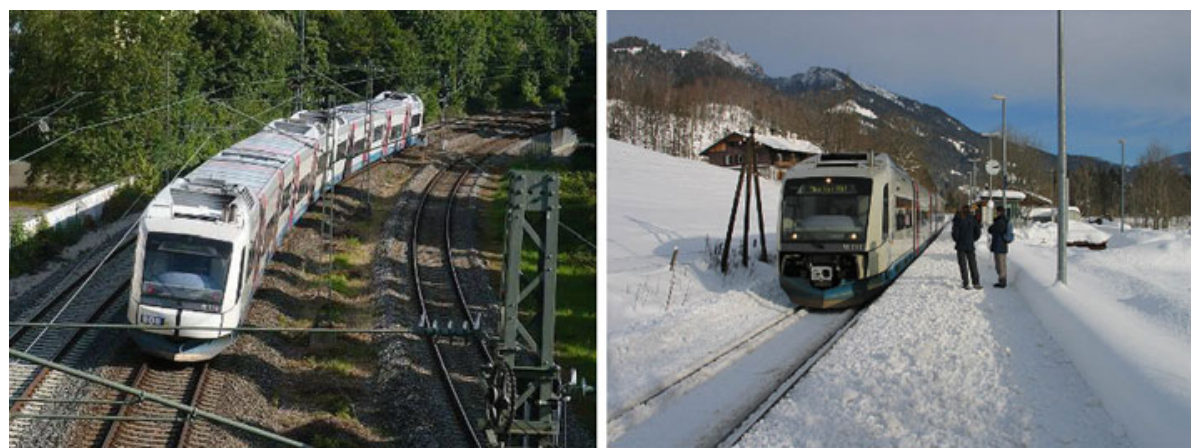

Fig. 3 A BOB train-set on the electrified two track line between Munich and Holzkirchen (left) and in the hilly alpine upland near Bayrischzell (right) [18]

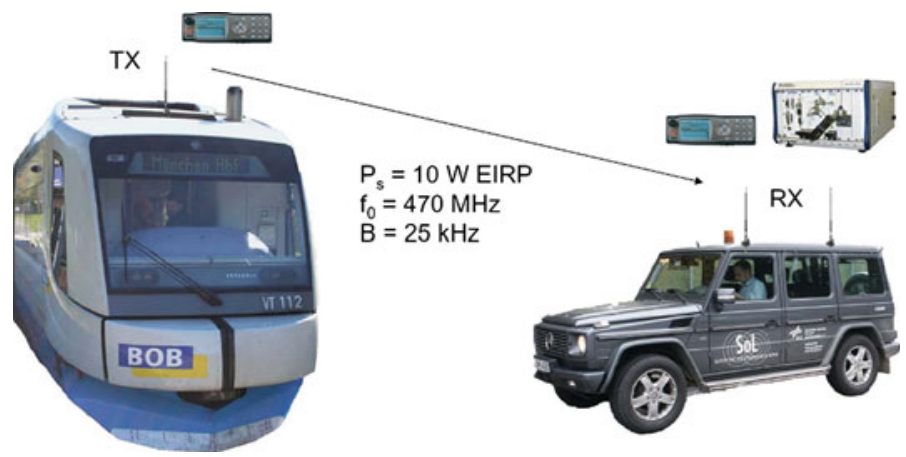

Fig. 4 Setup for the "train-to-train" channel measurement campaign: a car replaces the second train to allow for a variety of measurement scenarios at different locations

\subsection{Measurement Setup}

In regular operation the $\mathrm{BOB}$ train-sets meet at 3 distinct locations: At the stations in Holzkirchen and Schaftlach, where the train-sets are separated and connected, respectively, and at a point north of Holzkirchen, where 2 trains pass by each other on their way to and from Munich. In order to measure a variety of scenarios at many different locations along the network, we decided to use a car instead of a second train as receiving station for the measurements as depicted in Fig. 4. The car also allowed us to simulate hypothetical railway collisions, which would not be realizable with two trains during regular operations, because of safety regulations and the general railway network topology.

The transmitter was installed into one of the trains with the antenna mounted above the driver's cabin. The measurement signal was transmitted by a TETRA radio at a carrier frequency close to $470 \mathrm{MHz}$ with a power of $10 \mathrm{~W}$ EIRP, and had a bandwidth of $25 \mathrm{kHz}$. With a repetition rate of $1 \mathrm{~Hz}$ we transmitted a 150 bit SDS message containing traffic relevant information for the RCAS system as described in [5].

On the receiver side another TETRA radio recorded the successfully decoded messages. In addition the RFSA described in Sect. 4 was used to sample and record the complex received signal at a rate of $200 \mathrm{kHz}$ in the time domain as shown exemplarily in Fig. 5. According to our setup, the synchronization covers two frames (here 17 and 18), followed by the SDS data 


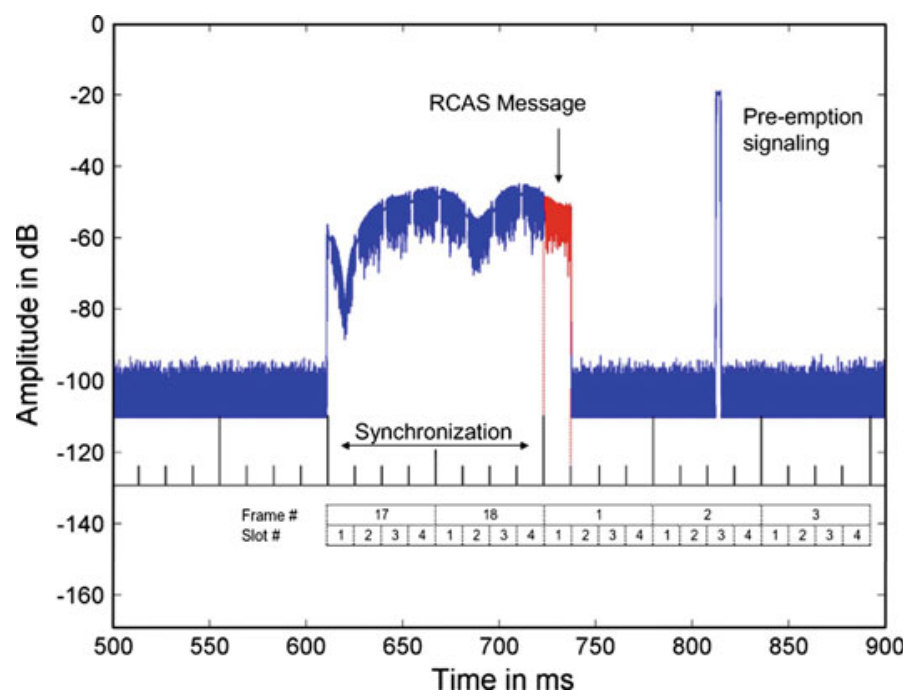

Fig. 5 Example of the recorded measurement signal (shown is the amplitude of the complex signal) during a pass by with moderate speed, clearly showing signal fading due to nearby multipath

in slot 1 of frame 1 . The pre-emption signaling in slot 3 of frame 2 in the example in Fig. 5 indicates the priority request for further transmissions. The example in Fig. 5 was recorded during a pass by at moderate speed, and it clearly shows the fading due to multipath. In the later analyses of the propagation channel characteristics the synchronization sequence is evaluated.

Both vehicles also carried a GPS receiver and stored PVT (Position, Velocity and Time) information during the measurements.

\subsection{Measurement Scenarios}

During the whole measurement campaign 26 scenarios were measured, collecting $16 \mathrm{~h}$ of data on more than 700 travelled kilometers. Two methods were applied alternatively:

- Static RX measurements: The car with the RX equipment was placed just aside the railway track or on a bridge above the track, and recorded the measurement signal while the train was approaching and leaving the measurement location.

- Dynamic RX measurements: The car was driven along roads just aside the railway track to simulate a moving train. Thus we could investigate hypothetical collision scenarios such as front, rear-end, and flank collisions with the approaching train.

Propagation conditions in railway environment, especially shadowing effects and multipath, may vary significantly depending on the local topology of the railway network. For example in stations the tracks are usually separated by platforms with roofs that might block the LOS (Line of Sight), while in shunting areas LOS is reasonable for larger distances, but it is usually accompanied by strong multipath from other vehicles and the particular environment. The speed and therefore the signal fading are significantly slower near stations than on the sections of the regional network between them. Urban, suburban and rural passages are expected to show different path loss characteristics, depending if there are houses, 
Fig. 6 Power spectral density of the TETRA synchronization burst that is used as measurement signal for the characterization of the train-to-train propagation channel

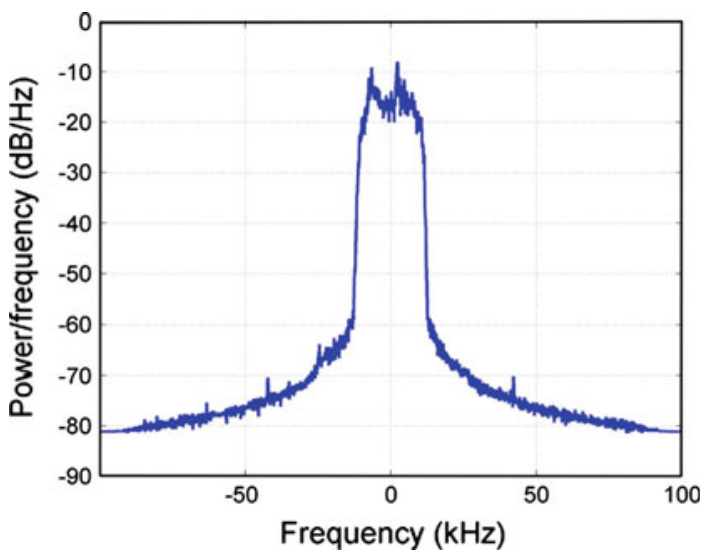

trees or slopes alongside the track. Thus measurements were conducted in the following environments:

- Urban main station (Munich central station)

- Suburban stations (Solln, Holzkirchen, Bad Tölz)

- Shunting area (near Munich central station)

- Tunnel (near Donnersberger Brücke)

- Forest and farmland passages (multiple locations)

- Hilly alpine upland (Bayrischzell, Lenggries, Tegernsee)

\subsection{Data Analysis}

Prior to the measurement campaign we made a calibration measurement, where we connected the TX radio output via a cable and an attenuator to the RFSA, recording the specific TETRA synchronization sequence $s(t)$ that is transmitted by the TX radio. The power spectral density of $s(t)$ can be seen in Fig. 6.

Distorted by the propagation channel and additional noise, the complex received signal $y(t)$ during the measurement campaign is given by

$$
y(t)=h(t) * s(t)+n(t),
$$

where $h(t)$ denotes the momentary channel impulse response and $n(t)$ is an additive white Gaussian noise (AWGN) signal. For AWGN the Maximum-Likelihood estimation of the channel impulse response is given by cross-correlation of the received signal $y(t)$ and the transmitted signal $s(t)$, or expressed in the frequency domain, the momentary channel frequency response $H(f)$ (power spectral density as function of Doppler frequency) is given by

$$
H(f)=Y(f) S^{*}(f) /|S(f)|^{2}=Y(f) / S(f),
$$

with $Y(f)$ and $S(f)$ being the Fourier transform of $y(t)$ and $s(t)$, respectively [19]. Assuming perfect synchronization between transmitter and receiver, $H(f)$ can be calculated from the DFT (Discrete Fourier Transform) of the sampled receive signal divided by the DFT of the transmit signal.

In our measurements the TX and RX stations were clocked by standard internal crystal oscillators, which causes an error when calculating $H(f)$. This error is mainly a slowly 

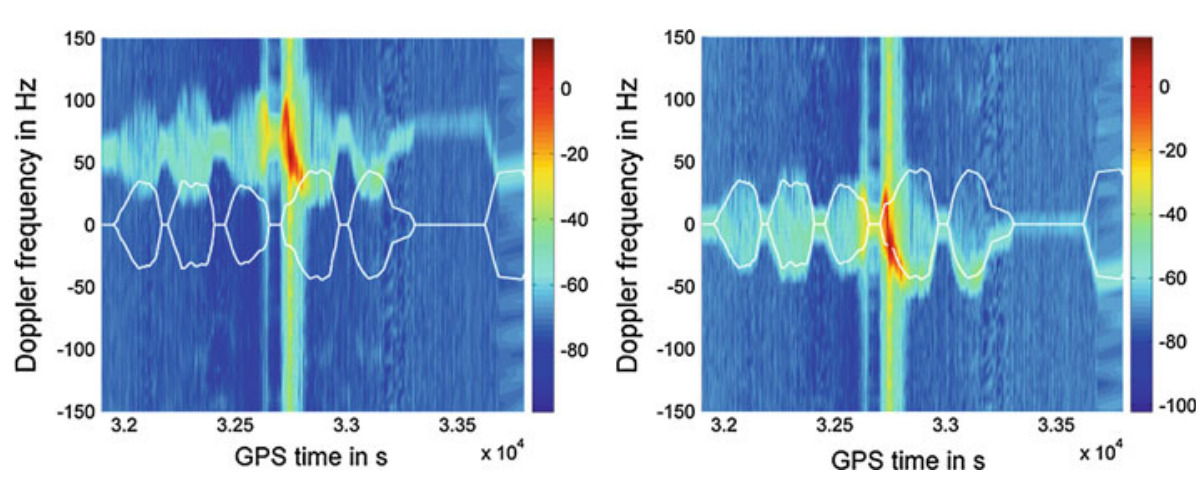

Fig. 7 Changes in the channel frequency response $H(f)$ in $\mathrm{dB}$ during a complete measurement run. The white lines indicate the maximum possible Doppler due to RX and TX movement. Left Offset due to RX and TX clock drift. Right Corrected clock drift offset

varying offset in Doppler due to drift changes of the oscillators. In the measurement data this offset can be easily determined, provided that PVT data of both stations is available. For example in sections when both, RX and TX were at a stop, the Doppler of the received signal shall be zero.

Figure 7 illustrates this correction. In the left plot we see the channel frequency response in $\mathrm{dB}$ over a complete measurement run that lasted more than $30 \mathrm{~min}$. Each second the received synchronization sequence was evaluated. During this measurement the receiver was static at one location. The white lines indicate the maximum possible Doppler according to the velocity of the transmitter. Hence, each stop of the train at a railway station is represented by a section where the white curves equal zero. The corrected data can be seen in the right plot of Fig. 7, after estimating the clock drift and removing the corresponding Doppler offset. It shows a very good match and clearly allows distinguishing between LOS and multipath components, as we will see in the following analysis. Note that the SNR is approximately $30 \mathrm{~dB}$ and that the frequency resolution in these plots is about $6 \mathrm{~Hz}$ due to the finite length of the synchronization sequence $s(t)$ and the sample rate of $200 \mathrm{kHz}$. Thus, according to Fig. 7 there seem to be spectral components outside the theoretical Doppler bandwidth. However, these are effects of the limitations of the sampling.

\subsubsection{Suburban Railway Station and Forest}

The data shown in Fig. 8 was recorded at the railway station in the small Bavarian town of Bad Tölz in a static RX measurement. The train approached from the south west, stopped at the station just $200 \mathrm{~m}$ in front of the RX position, which is marked by the yellow triangle in Fig. 9, and continued passing by the RX position, leaving the town into some minor forest passages. The TX track is indicated with red diamonds, the size of which show the HDOP (Horizontal Dilution of Precision) recorded by the GPS receiver. It can be seen, that the HDOP significantly increases in forest sections, and even when passing beyond a street (see right top corner of Fig. 9).

When the train approached the station between time stamps 100-150s (the last $700 \mathrm{~m}$ before the station), we can see a clear increase in power in Fig. 8. The direct wave components are at approximately $+20 \mathrm{~Hz}$, but there are as well very strong components from scatterers that reach almost the same power level. Please note that the power in Fig. 8 is normalized to the hypothetical FSL (Free Space Loss), i.e. we only show the additional 
Fig. 8 Measured channel frequency response in $\mathrm{dB}$ at the railway station in Bad Tölz (power normalized)
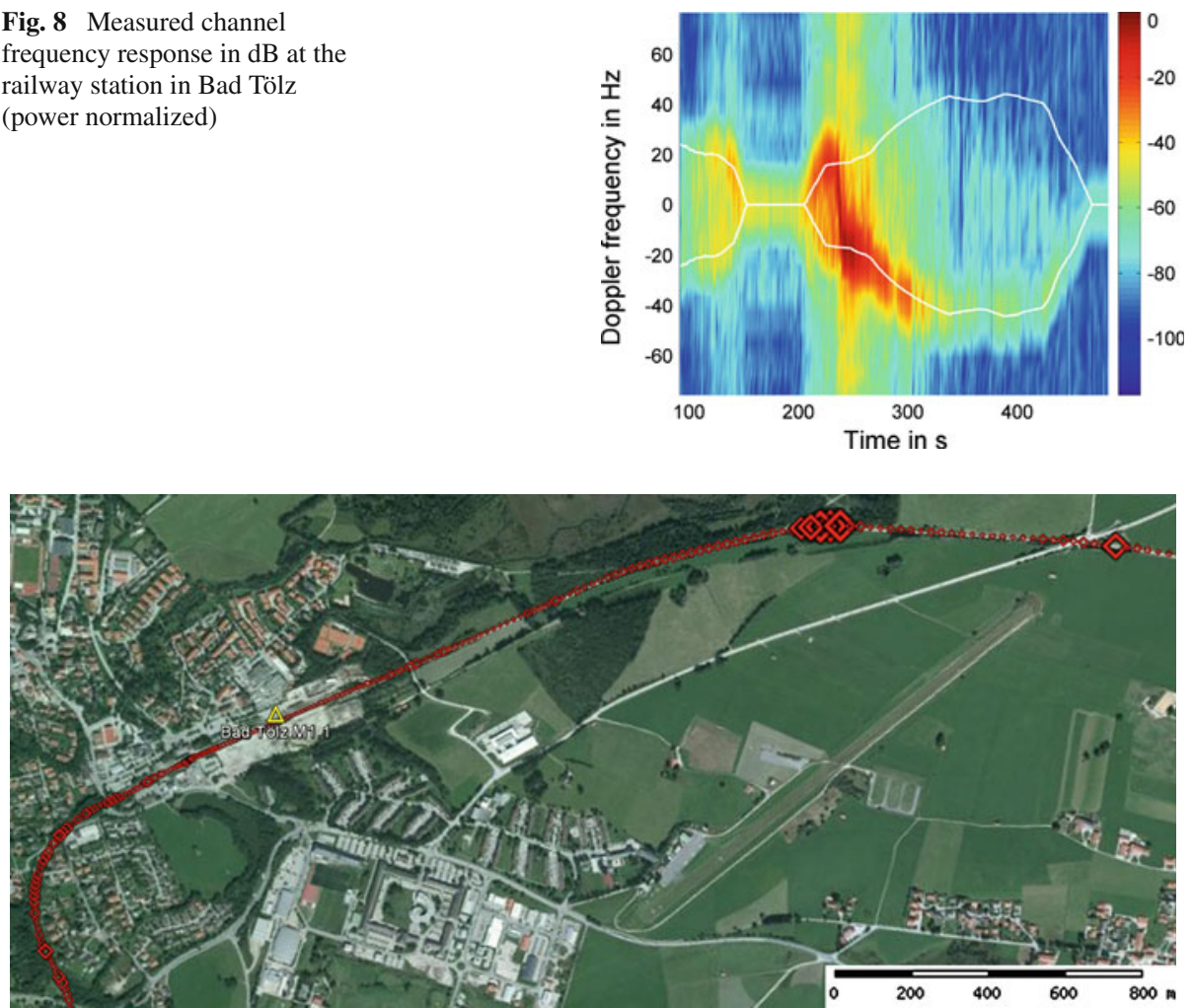

Fig. 9 Suburban measurement scenario in the vicinity of the railway station in Bad Tölz. Red diamonds indicate the route of the train; the yellow triangle shows the static RX position

loss due to diffraction and reflection. In other words, the red parts with $0 \mathrm{~dB}$ refer to LOS condition.

During the halt from 150 to $200 \mathrm{~s}$, we can see a characteristic lower power caused by the roofs on the platforms, which block the LOS signal. Immediately after leaving the station the LOS path appears, quickly changing its Doppler from +20 to $-20 \mathrm{~Hz}$ when the train passes by the receiver at time stamp 240. In the whole area of the station strong multipath was present from different directions causing a maximum Doppler spread, until the LOS signal vanished as the train entered a forest passage after $300 \mathrm{~s}$ from start (approximately $1.2 \mathrm{~km}$ past the station), where only scattering from objects and vegetation on both sides of the track with a Doppler frequency around zero remains beside the diffracted direct path with negative Doppler.

\subsubsection{Tunnel in Urban Environment}

The measurement result shown in Fig. 10 was recorded near the Munich central station. Again the receiver was static at a location very close to the railway track (see yellow triangle in Fig. 12). The train started at the Munich central station and had its first stop just beyond the bridge "Donnersberger Brücke" in the upper part of the image. Right after this stop, the train entered a tunnel that crosses under other tracks, allowing to directly going south passing 
Fig. 10 Measured channel frequency response in $\mathrm{dB}$ in the urban environment near the tunnel at Donnersberger Brücke in Munich

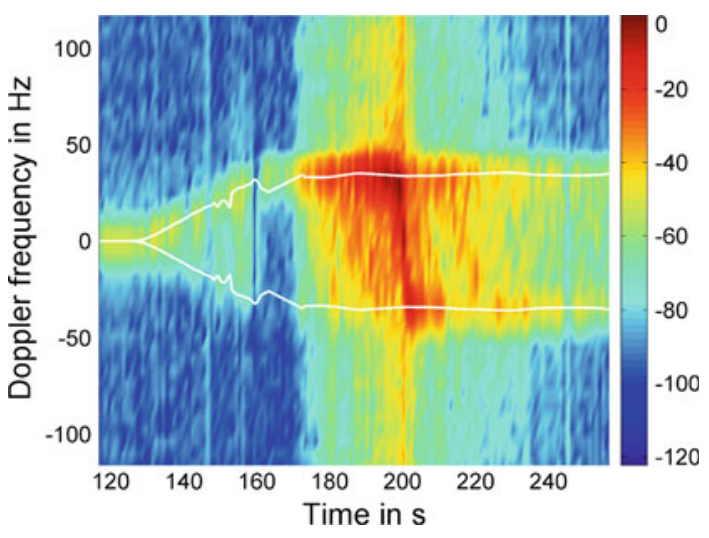

Fig. 11 Measured relative power in urban environment near the tunnel in Munich, showing the evolution of path loss in this scenario

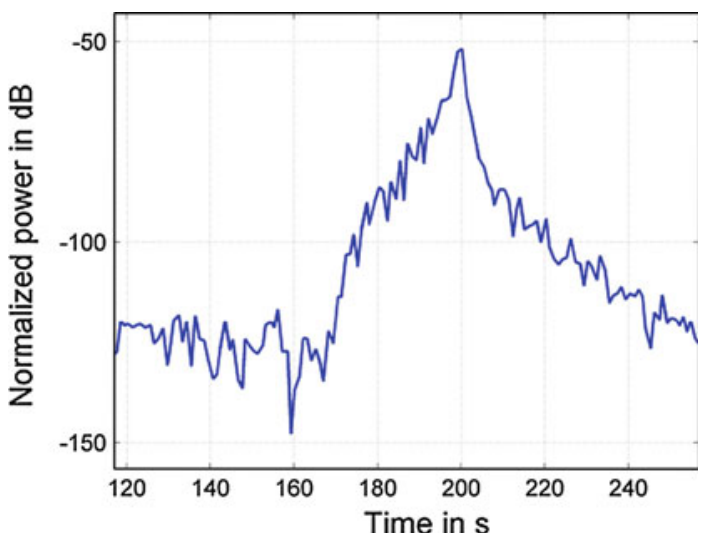

by the receiver location. This tunnel is about $280 \mathrm{~m}$ long and is indicated by the white line in Fig. 12. The red diamonds again indicate the TX track, showing increased HDOP values, when passing under the bridge and at the tunnel entrance and exit, where less GPS signals are tracked.

The measured channel frequency response of this scenario is shown in Fig. 10. The train starts to leave Donnersberger Brücke station at time stamp 130. Between time stamp 140 and 150 some fading is notable on the direct signal at the moments when the antenna passed behind the abutments of the bridge. At the same time right under the bridge the GPS velocity measurements become erroneous, which can be clearly seen in the white line that marks the maximum possible Doppler. Soon after that, at time stamp 160, the train-set with the antenna entered the tunnel via a ramp. A short significant signal loss is visible at just that moment and all the multipath components disappear. It is very interesting to note that in the following $10 \mathrm{~s}$, when the train passes through the tunnel, the received signal level is almost as high as before. This can be best observed in Fig. 11, which shows the evolution of the normalized received power for the same section of the measurement. Note that this corresponds to the evolution of total path loss including the FSL (Fig. 12).

After the train exits the tunnel at about timestamp 175, the signal strength increases rapidly, as we begin to have LOS condition. At time stamp 200 the train passes by the RX station heading further south. Very strong multipath exists in this urban railway environment (see 


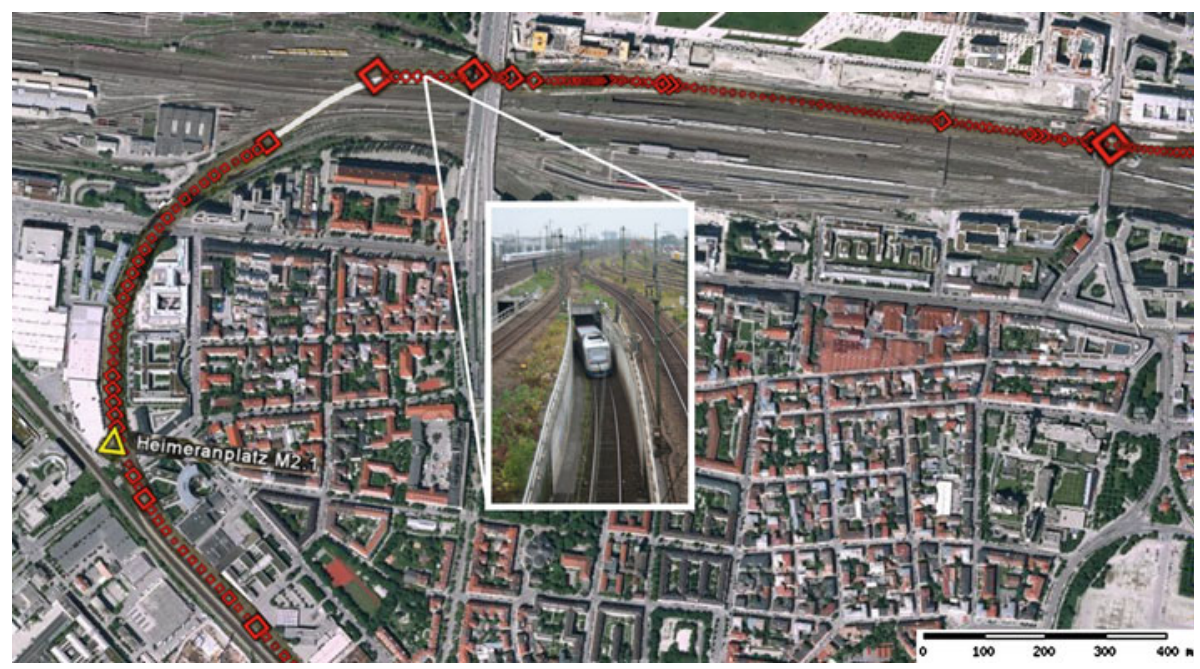

Fig. 12 Urban measurement scenario near Donnersberger Brücke in the city center of Munich; the white line marks the tunnel that is used to cross multiple other tracks

Fig. 13 Comparison of theoretical path loss model and the train-to-train measurement result

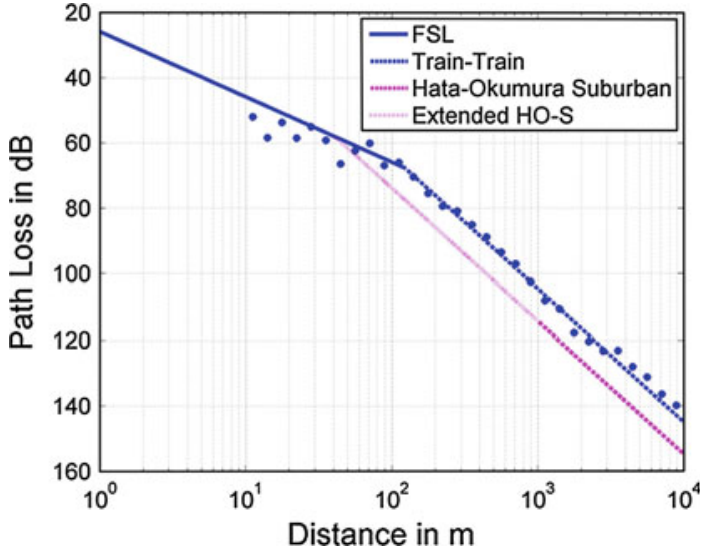

Fig. 10), which tends to show a typical Jakes spectrum characteristic [20], as the transmitter departs from the receiver location after time stamp 220 s, already $500 \mathrm{~m}$ past the RX position.

\section{Channel Characterization}

\subsection{Path Loss}

In [21] existing channel models for terrestrial communication and their applicability to trainto-train scenarios are discussed. As a result Hata-Okumura models were proposed for modeling the path loss in different railway environments. Hereby the suburban characteristic best fits the mix of scenarios that we measured during our campaign. In Fig. 13 we compare the averaged path loss of the entire measurement data (blue dots) with the theoretical model—the 
Table 3 Comparison of path loss model parameters

Fig. 14 Example of slow fading in an urban measurement scenario, expressed as mean received signal power normalized to the path loss model

\begin{tabular}{lll}
\hline Parameter & Train-to-train & $\begin{array}{l}\text { Extended Hata } \\
\text { Okumura-suburban }\end{array}$ \\
\hline Mean LOS range & $125.9 \mathrm{~m}$ & $40 \mathrm{~m}$ \\
Path loss & 4.04 & 4 \\
exponent (non LOS) & & \\
\hline
\end{tabular}

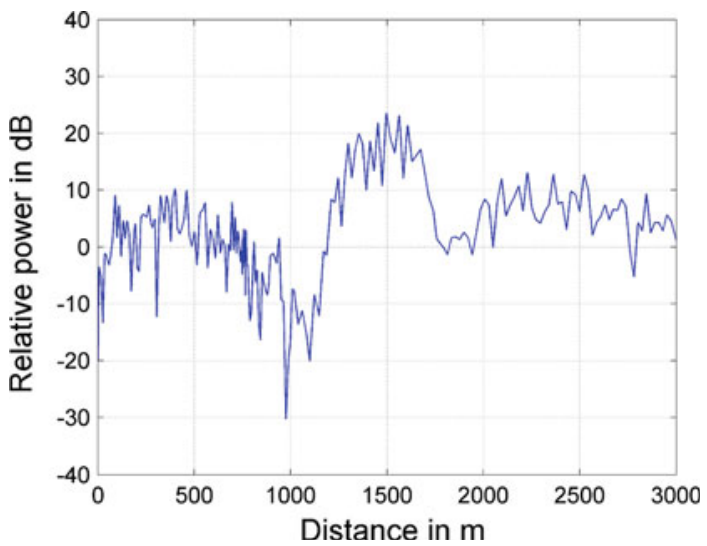

Hata-Okumura suburban path loss model [22] that was evaluated for $f_{0}=470 \mathrm{MHz}$, and a receive and transmit antenna height $h_{r}=h_{t}=4.8 \mathrm{~m}$. First of all, we note that in case of direct train-to-train communication the LOS condition is maintained for larger distances than in other terrestrial applications, typically more than $100 \mathrm{~m}$. While the exponential decay is in the expected order, the path loss for non LOS condition was observed to be $10 \mathrm{~dB}$ lower than according to the model, which in fact results in almost a $100 \%$ increase, i.e. a doubling of the communication range. Table 3 summarizes the characteristic of both models.

\subsection{Fading}

For the envisaged safety related VANET applications one of the main criteria is the distribution of communication outages due to fading. Slow fading, which is typically imposed by shadowing of large objects or even forests or hills, can lead to the subsequent loss of transmitted messages between the trains. To investigate this characteristic we analyzed the mean power of the received signal sequences similar to the example depicted in Fig. 5. We applied the train-to-train path loss model just described to determine the slow fading power variation. An example from an urban measurement scenario is shown in Fig. 14, where the mean received signal power normalized to the path loss model is plotted vs. the distance between RX and TX.

In terrestrial communication the slow fading characteristic is typically described by means of the correlation distance, i.e. the distance where the Auto-Correlation Function (ACF) drops below 0.5. For suburban environments in base station to mobile scenarios, the ACF was determined and modeled in [23] with a correlation distance of approximately $400 \mathrm{~m}$.

In case of direct train-to-train communication the autocorrelation of slow fading can vary in a much wider range, as the analysis of our measurement data in Fig. 15 shows. The correlation distance changes between 200 and $2,800 \mathrm{~m}$ in the various scenarios. The lowest 
Fig. 15 Autocorrelation function of slow fading in train-to-train communication scenarios

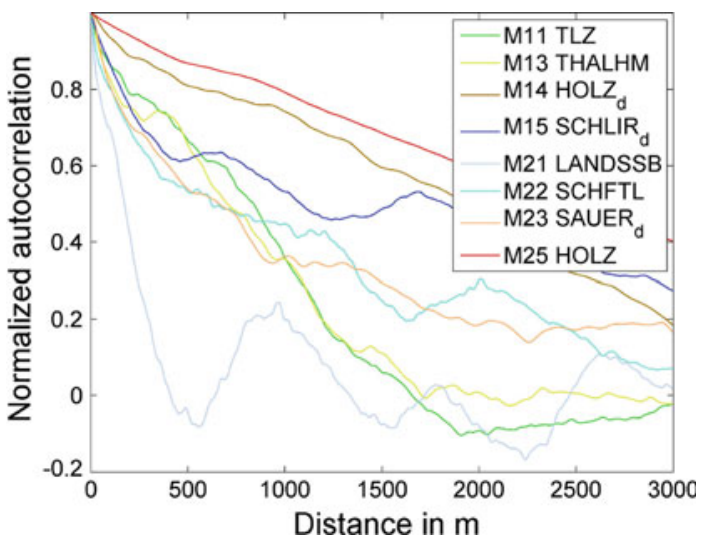

Table 4 Slow fading characteristics in Train-to-train communication compared to conventional base station to mobile communication scenarios

\begin{tabular}{ll}
\hline Scenario & Correlation distance \\
\hline Base station to mobile station (suburban) & $\sim 400 \mathrm{~m}$ \\
Train-to-train (suburban) & $\sim 1,000 \mathrm{~m}$ \\
Train-to-train (urban-rural) & $200-2,800 \mathrm{~m}$
\end{tabular}

correlation was observed in urban environment (M21), whereas the largest values occurred in straight rural sections (M14 \& M25). All other curves shown in Fig. 15 are depicting different scenarios, such as station, forest, farmland or hilly terrain in suburban and rural environments, all showing correlation distances in the order of $1 \mathrm{~km}$. Table 4 summarizes the new findings.

In contrast to slow fading, fast fading is the result of multipath propagation. The Doppler spectra in Sect. 5.4 gave evidence of heavy multipath in the railway environment. The effect of this fast fading is an increased bit error rate, which leads to higher probability of message losses, as we will see in Sect. 7.2. Values for Doppler spread, delay spread, Rice and Rayleigh parameters, respectively, have been determined in measurements before and are summarized in [21]. A coarse look on our data didn't reveal major differences.

\section{TETRA Performance Analysis}

The analysis on the received signal in the previous section showed that the direct trainto-train propagation conditions are advantageous in terms of path loss. On the other hand, severe fading due to strong multipath in the railway environment was observed, that affects the performance of SDS transmission in DMO.

\subsection{Communication Range}

A major requirement in safety applications like RCAS is to achieve the necessary communication range. In case of low user density in VANETs, the forwarding of data transmissions on the network layer in a multi-hop way is not possible. Thus, without any infrastructure, two approaching trains need to be able to exceed the sum of their maximum braking distances within their communication range. For extended regional railroad services with speeds of up 
Fig. 16 Probability density function of the communication range with TETRA DMO-SDS in direct train-to-train communication scenarios

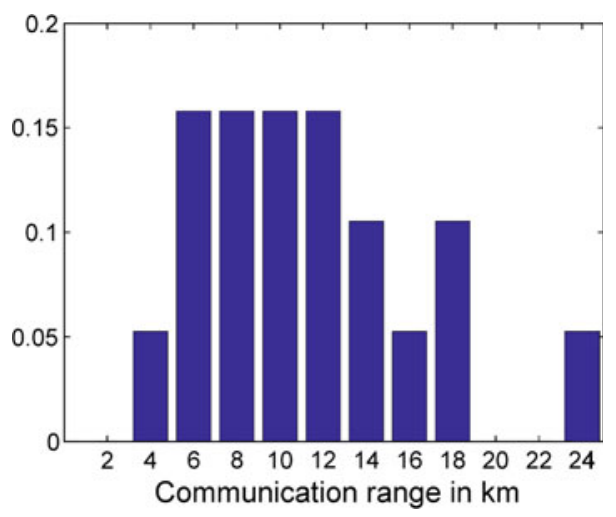

Table 5 Maximum permissible MS receiver message erasure rates (MER) at reference sensitivity level in DMO [11]

\begin{tabular}{llll}
\hline Logical channel & Propagation model & Reference level $(\mathrm{dBm})$ & MER $(\%)$ \\
\hline $\mathrm{SCH} / \mathrm{F}$ & DU50 & -103 & 5.40 \\
$\mathrm{SCH} / \mathrm{F}$ & DR50 & -103 & 8.00 \\
$\mathrm{SCH} / \mathrm{F}$ & STATIC & -112 & 9.00 \\
\hline
\end{tabular}

to $200 \mathrm{~km} / \mathrm{h}$ in some exceptional cases, the maximum braking distance of a single train is below $1,500 \mathrm{~m}$.

The TETRA and GPS recordings allowed us to determine the distance between RX and TX, when the first SDS transmission succeeded during each approach of the train. The distribution of this communication range is depicted in Fig. 16. The minimum range was $4.3 \mathrm{~km}$, thus in each of the 19 scenarios where we approached from a distance of at least $10 \mathrm{~km}$-including all propagation critical terrain sections along the network-the required $3 \mathrm{~km}$ distance was exceeded.

\subsection{Message Erasure Rate}

The TETRA standard defines in [11] the receiver performance measures at reference sensitivity levels according to the logical channels. For MSs in DMO the static and dynamic reference sensitivity levels are given as -112 and $-103 \mathrm{dBm}$, respectively.

The logical channel used for SDS-type 4 transmission in DMO is the Full-slot Signaling CHannel (SCH/F). It is used for both, the synchronization burst (DSB) transmission and for the normal burst (DNB) transmission, which carries the user defined data. The maximum permissible MS receiver Message Erasure Rates (MER) for the SCH/F at the reference sensitivity levels are given in Table 5. These measures were derived according to simulations during the TETRA design phase taking AWGN conditions and the specified TETRA channel models into account, where DU50 and DR50 denote models for Urban Area and Rural Area, respectively, and a vehicle speed of $50 \mathrm{~km} / \mathrm{h}$ [6].

In order to correctly receive the user data on the DNB, the synchronization of the TETRA receiver in DMO has to be established. The synchronization performance is defined in terms of the Probability of synchronization burst ACQuisition (PACQ) of detecting a DSB and correctly decoding the $\mathrm{SCH}$ information for the condition where the MS is listening on the 
Table 6 MS receiver performance requirement (probability of correct detection and decoding) for synchronization burst acquisition

\begin{tabular}{llll}
\hline Logical channel & Propagation model & Reference level & PACQ \\
\hline $\mathrm{SCH} / \mathrm{F}$ & DR50 & $-103 \mathrm{dBm}$ & $80 \%$ \\
\hline
\end{tabular}

frequency while the DSB is transmitted. In [11] the required PACQ is defined for the dynamic receiver sensitivity level of $-103 \mathrm{dBm}$ given propagation conditions according to the DR50 channel model (Table 6).

In our train-to-train measurement campaign we were not able to determine and verify the single measures for the logical channel, but we determined the overall synchronization and data reception performance under these special propagation conditions. Figure 17 illustrates the result. Plotted is the MER with respect to the transmitted SDS messages. Limited by the sensitivity of the RFSA the detectable received power level was approximately $-112 \mathrm{dBm}$. For static situations in the measurement data the MER did not largely increase down to that level, but for the dynamic case a clear performance degradation is visible: The MER starts to increase significantly for power levels below $-103 \mathrm{dBm}$ and Doppler frequencies of more than $10 \mathrm{~Hz}$. Given our measurement frequency of $470 \mathrm{MHz}$, a vehicle movement of $50 \mathrm{~km} / \mathrm{h}$ corresponds to a Doppler of

$$
f_{D}=\frac{v}{c} f_{0}=\frac{(50 / 3.6) \mathrm{m} / \mathrm{s}}{3 \cdot 10^{8} \mathrm{~m} / \mathrm{s}} 470 \cdot 10^{6} \mathrm{~Hz}=21.8 \mathrm{~Hz} .
$$

For our specific SDS setup with two DSB frames and without data re-transmission, the expected performance for a dynamic situation at reference sensitivity with $50 \mathrm{~km} / \mathrm{h}$ in rural environment given the requirements in the tables above is

$$
\mathrm{MER}_{\mathrm{DMO}-\mathrm{SDS}}=(1-\mathrm{PACQ})^{2}+\mathrm{MER}_{\mathrm{DR} 50}=(1-0.8)^{2}+0.08=12 \%
$$

Although the measured MER for DMO-SDS transmission in Fig. 17 follows roughly the expectations from the standard, there are a few important findings. First of all we see that the MER often exceeds $12 \%$ at low signal power levels in the railway environment. For increasing Doppler, the MER increases and reaches values of up to $40 \%$ at reference sensitivity level. Moreover, MER levels of up to $40 \%$ also occurred at medium Doppler for power levels above the reference level. On the other hand, the static performance limit was observed to be below the reference level of $-112 \mathrm{dBm}$. Nevertheless, 10-30\% MER occurred up to power levels of $85 \mathrm{dBm}$ even in the static case. Static situations in our measurements only happened when the train was at a halt in a station. In such situations strong multipath is typical as we saw in the Doppler spectra in Figs. 8 and 10. In fact, even when transmitter and receiver were static, the movement of other railway or road vehicles in the vicinity of stations can cause fast fading. Heavy multipath near stations, where the train in general moves slower than in the rural sections, also explains why the MER tends to be higher than calculated in (4) at Doppler frequencies between 10 and $40 \mathrm{~Hz}$ even at power levels above the reference sensitivity.

\subsection{MER for Multi-User Access}

In addition to propagation related SDS transmission failures, messages can also get lost when multiple radios are transmitting their traffic information on a common channel. Although the exposed terminal problem does not exist in beaconing VANETs, the other typical sources 
Fig. 17 MER for DMO-SDS

transmission in direct

train-to-train communication scenarios

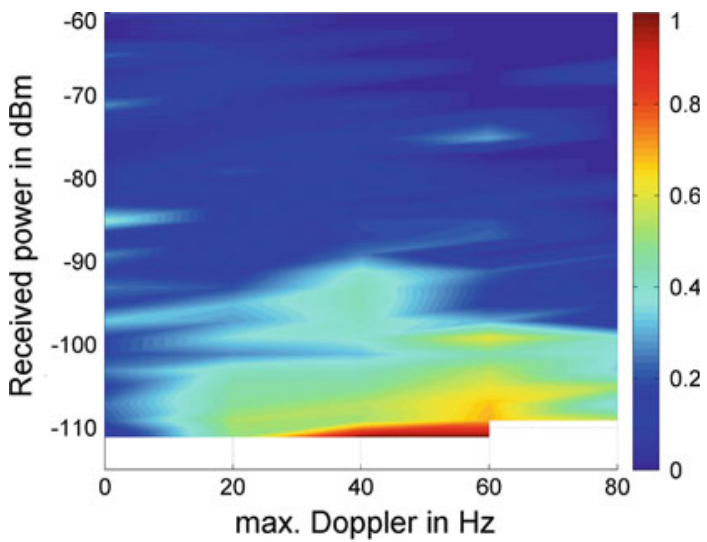

for data traffic collisions, i.e. the hidden terminal problem, still exists. The performance in this multi-broadcast scenario is limited by the Media Access Control (MAC) implemented in a system. MAC in TETRA DMO was not designed for such scenarios but incorporates some techniques that allow sustaining service in high loaded or even overloaded condition, although with limited performance.

The necessary rate for broadcasting new position information in VANETs is usually related to the velocity of vehicles. While a train with $200 \mathrm{~km} / \mathrm{h}$ loses more than $50 \mathrm{~m}$ of potential braking distance per second if no information from a potential collision opponent is received, a transmission interval of 5-10 s can be sufficient in low speed sections. For the measurement campaign a rate of $1 \mathrm{SDS} / \mathrm{s}$ was chosen, which already leads to a MER of approximately $4 \%$ (see floor in Fig. 17). The end-to-end transmission delay varied between 200 and $700 \mathrm{~ms}$. Both measures were confirmed by static tests at short distance and negligible multipath in an office environment. In a further test, with a transmission interval of $2 \mathrm{~s}$ for a single station $(0.5 \mathrm{~Hz})$, the MER was observed at $0.2 \%$, and the end-to-end transmission delay including the serial port communication on the PEI with the TX and RX radios was constant at $200 \mathrm{~ms}$.

In order to assess the performance in multi-broadcast situations we performed another test, where three TETRA stations were concurrently accessing the channel to transmit their data. They were placed in short range to each other, i.e. a closed network configuration where no hidden terminal problem occurs. The result is shown in Fig. 18. For the case that each station transmits at a rate of $1 \mathrm{~Hz}$, the overall MER is less than $6 \%$, which means a degradation of $2 \%$ compared to the case that only one station is transmitting as it was the case throughout our railway measurement campaign. When the transmission rate is increased to $4 \mathrm{SDS} / \mathrm{s}$, a significant increase to $10 \%$ in the MER can be observed.

With respect to the railway scenarios we are investigating, the transmission of $4 \mathrm{SDS} / \mathrm{s}$ from different stations is reasonable. This scales for scenarios of 2-5 trains on high-speed sections with long braking distances broadcasting at $0.5-1 \mathrm{~Hz}$, as well as for shunting and station operations of 5-15 trains at low to very low speed broadcasting at $0.125-0.25 \mathrm{~Hz}$ as stated in [3].

\subsection{Update Distance}

While fast fading and inter-symbol interference are causing single messages to be lost, the special slow fading characteristics in the train-to-train communication environment can cause long outages, where a series of SDS transmission failures occur. For applications like RCAS, 
Fig. 18 MER for concurrent DMO-SDS transmission from 3 MSs in static office environment

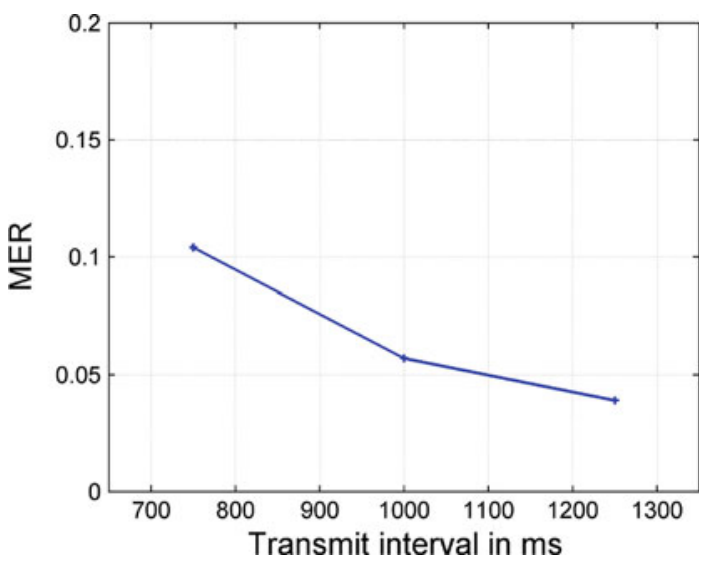

Fig. 19 Complementary cumulative distribution functions of the "update distance": showing the probability that a given value of the "update distance" is exceeded when RX and TX are closer than 5,3 or $2 \mathrm{~km}$, respectively, for two different channel loads

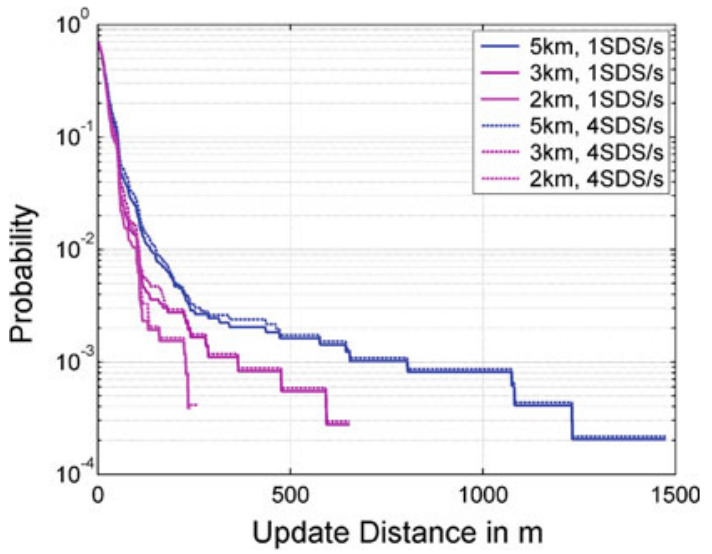

the probability of receiving a status update from nearby trains within a given time or travelled distance, respectively, is a major QoS criterion [5].

We define the update distance as the distance a receiver and a specific transmitter node move alongside the railway track, during the time of two consecutive successful receptions of messages from this transmitter at the receiver. The Complementary Cumulative Distribution Function (CCDF) of the update distance calculated from data recordings of the whole measurement campaign is plotted as solid lines in Fig. 19.

The CCDF defines the probability that a given value of the "update distance" is exceeded. For instance when analyzing the communication between TX and RX while they were less than $5 \mathrm{~km}$ apart, the blue curve shows that the probability of not receiving a status update over the next e.g. $500 \mathrm{~m}=$ "update distance" (travelled distance of both, TX and RX) is approximately $0.2 \%$. The closer TX and RX are, the shorter are communication outages, which results in lower probabilities as shown by the other solid curves, e.g. when TX and $\mathrm{RX}$ were less than $3 \mathrm{~km}$ apart an outage for more than $500 \mathrm{~m}$ occurred with a probability of only $0.05 \%$.

The statistics shown by the solid lines in Fig. 19 do not take possible effects due to multiuser access into account. Other than in our office tests, in case of dynamic VANETs the MAC mechanisms could fail to some extent. On the other hand, due to path loss, messages from 
near trains can be received even when interfered from trains at larger distances. Performance degradation or even partial improvement depending on the channel load is likely. However, for moderate load minor changes are expected, since the MER due to message collisions on the channel (see decay in Fig. 18) is often lower than the observed MER in Fig. 17, especially at large distances (low receive power levels). Assuming an overall increase of the MER by $6 \%$ when increasing the load from 1SDS/s to 4 SDS/s, the dashed lines in Fig. 19 show, that this results in only a minor performance degradation of the CCDF of the update distance.

\section{Conclusion}

In the presented study we analyzed the performance of TETRA short data service in direct mode operation under railway operational conditions. Data from comprehensive measurements shows that TETRA can be adopted for beaconing VANET in the railway environment, and that applications requiring reliable direct train-to-train communication can benefit from the specific environmental conditions. Due to the layout of railway lines and the typical character of passages through urban, suburban and rural areas, the wave propagation for UHF frequencies is significantly better than in other mobile-to-mobile scenarios. Line of Sight (LOS) condition is typically maintained for distances of more than $100 \mathrm{~m}$ and the path loss was found to be $10 \mathrm{~dB}$ lower than expected from theoretical models, resulting in a communication range of at least $4 \mathrm{~km}$ with $10 \mathrm{~W}$ transmit power.

As a drawback, strong multipath around stations and shunting areas was observed leading partly to higher message erasure rates than according to simulation results with the reference channel models in the ETSI TETRA standard. Moreover, due to the low antenna levels increased shadowing of e.g. forests causes slow fading, which can lead to the subsequent loss of transmitted messages. The spatial correlation of the analyzed slow fading in the investigated regional railway network was observed to vary in a large range, depending on the environment and on how straight a railway section is.

Complementary to the measurement of propagation related effects we performed laboratory tests to evaluate the TETRA SDS-DMO performance in case of multi-user access, with respect to safety related applications. Reasonable good performance could be proven for a rate of 4SDS/s exchanged among three devices in a group call. End-to-end transmission delay was measured to be below $700 \mathrm{~ms}$. We introduced the update distance as measure to assess the performance of SDS exchange among mobile stations in a dynamic vehicular ad-hoc communication network. The resulting statistics show that a frequent and reliable traffic information update can be achieved even under higher load with multi-user access.

For applications like the railway collision avoidance system RCAS, conclusions on the quality of service can be given. We have seen that for higher velocities the train routes are usually straighter and the resulting longer breaking distances are likely to be covered by the enhanced communication range. In fact, an unexpected high margin for timely warning of train drivers in case of a collision threat from successfully received RCAS data during the measurement campaign was observed. Even in critical environments with obstacles like hills, or in case of a tunnel, the performance of TETRA SDS-DMO messaging could be proved to be highly reliable.

Acknowledgments The authors express their thanks to the management and staff of the "Bayerische Oberlandbahn" for their help and cooperation in performing the measurements, which led to a unique collection of data. 


\section{References}

1. Strang, T., Meyer zu Hörste, M., \& Gu, X. (2006). A Railway collision avoidance system exploiting ad-hoc inter-vehicle communications and Galileo. In Proceedings, 13th world congress on intelligent transportation systems, London, UK, October 8-12.

2. http://www.collision-avoidance.org/rcas. Accessed 8 May 2012.

3. Lehner, A., Strang, T., \& Rico García, C. (2008). A reliable surveillance strategy for an autonomous rail collision avoidance system. In Proceedings of the 15th ITS world congress, New York, USA, November 16-20.

4. Rico García, C., Lehner, A., Robertson, P., \& Strang, T. (2010). Performance of MAC protocols in beaconing mobile ad-hoc multibroadcast networks. In Proceedings of the third international workshop on multiple access communications MACOM, Barcelona, Spain: Springer, September 13-14, ISBN 978-3-642-15427-0.

5. Lehner, A., Rico García, C., Wige, E., \& Strang, T. (2009). A multi-broadcast communication system for high dynamic vehicular ad-hoc networks. In Proceedings of the first IEEE vehicular networking conference IEEE VNC, Tokio, Japan, October 28-30.

6. ETSI EN 300 392-2 V3.2.1. (2007-2009). Terrestrial Trunked Radio (TETRA); Voice plus Data $(\mathrm{V}+\mathrm{D})$; Part 2: Air Interface (AI).

7. Lienard, M., \& Degauque, P. (1998). Propagation in wide tunnels at $2 \mathrm{GHz}$ : A statistical analysis. IEEE Transactions on Vehicular Technology, 47(4).

8. Rico García, C., Strang, T., \& Lehner, A. (2008). A location-based MAC protocol for safety-of-life vehicle-to-vehicle communication. In C. A., Brebbia, J., Allan, E., Arias, C. J., Goodman, A. F., Rumsey, G., Sciutto, et al. (Hrsg.), Eleventh international conference on computer system design and operations in the railway and other transit systems COMPRAIL 2008, WIT transactions on the built environment (No. 103, pp. 305-314). Toledo, Spain: Wit Press, ISBN 978-1-84564-126-9, ISSN 1746-4498, September 15-17.

9. ETSI EN 300 396-1. (1998-2003). Terrestrial Trunked Radio (TETRA); Technical requirements for Direct Mode Operation (DMO); Part 1: General network design.

10. ETSI EN 300 396-3 V1.3.1. (2006-2008). Terrestrial Trunked Radio (TETRA); Technical requirements for Direct Mode Operation (DMO); Part 3: Mobile Station to Mobile Station (MS-MS) Air Interface (AI) protocol.

11. ETSI EN 300 396-2 V1.3.1. (2006-2009). Terrestrial Trunked Radio (TETRA); Technical requirements for Direct Mode Operation (DMO); Part 2: Radio aspects.

12. Dunlop, J., Girma, D., \& Irvine, J. (2000). Digital mobile communications and the TETRA system. Chichester: Wiley.

13. Stavroulakis, P. T. (2007). Errestrial trunked radio-TETRA—a global security tool, series: Signals and communication technology, XVI, p. 302, Springer, ISBN: 978-3-540-71190-2.

14. ETSI EN 300 392-5 V2.2.1. (2010-07). Terrestrial Trunked Radio (TETRA); Voice plus Data (V+D) and Direct Mode Operation (DMO); Part 5: Peripheral Equipment Interface (PEI).

15. Mills, D., et al. (June 2010). RFC 5905 network time protocol version 4: Protocol and algorithms specification.

16. TETRA Interoperability Certification, http://www.tetramou.com/. Accessed 8 May 2012.

17. http://www.bayerische-oberlandbahn.de. Accessed 8 May 2012.

18. http://en.wikipedia.org/wiki/Bayerische_Oberlandbahn. Accessed 8 May 2012.

19. Parsons, J. D. (2000). The mobile radio propagation channel (2nd edn). London: Wiley.

20. Jakes, W. C. (1974). Microwave mobile communications. New York: Wiley.

21. Rico García, C., Lehner, A., \& Strang, T. (2008). Channel model for train to train communication using the $400 \mathrm{MHz}$ band. In V., Srinivasan (Hrsg.) IEEE 67th vehicular technology conference VTC spring, pp. 3082-3086, Singapore: IEEE Conference express Publishing, ISBN 978-1-4244-1645-5, ISSN 1550-2252, May 11-14.

22. Proakis, J. G. (2001). Digital communications (4th edn). New York: McGraw Hill.

23. Gudmundson, M. (1991). Correlation model for shadow fading in mobile radio systems. Electronic Letters, 27(33), 2145-2146, ISSN: 0013-5194. 


\section{Author Biographies}
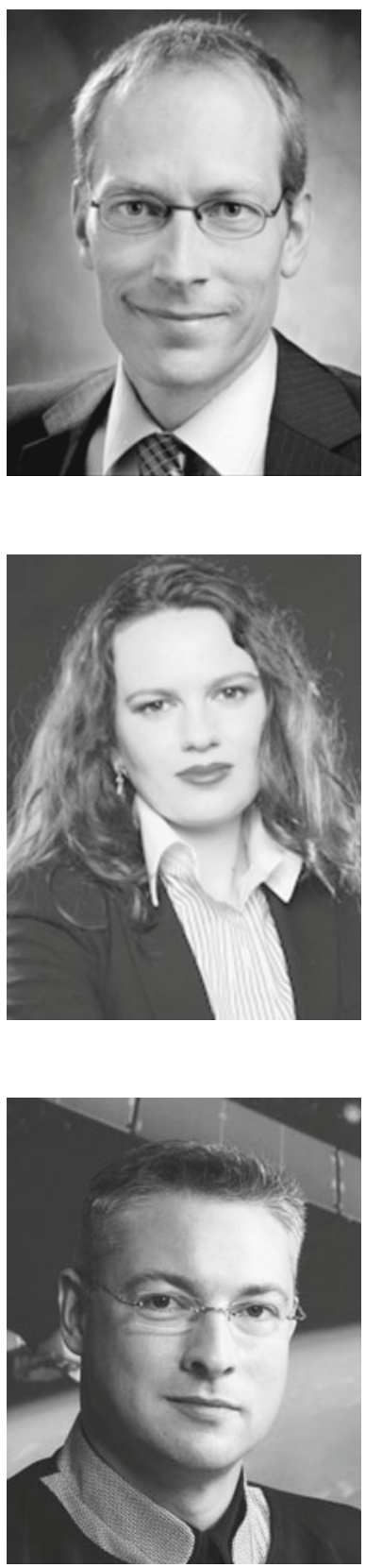

Andreas Lehner was born in Gmunden, Austria. He received the Dipl. Ing. degree in Mechatronics from the Johannes Kepler University in Linz in 2001 and a Ph.D. in Electrical Engineering from the University Erlangen-Nuremberg in 2007. Currently he is a senior research scientist at the Institute for Communications and Navigation at the German Aerospace Center DLR. His research and project work focuses on safety systems in transportation, the design of vehicle-to-vehicle communication systems, and on the characterization and analysis of multipath and interference effects in satellite navigation and communication systems.

Cristina Rico García was born in San-Sebastián, Spain. She pursued her studies at the University of Málaga, Politécnica de Valencia and Universität Ulm and obtained her final degree (Spanish Master equivalent) in Electrical Engineering in 2006. She has worked in different fields like speech recognition, Satellite Navigation and Communications. In 2006 she joined the German Aerospace Center (DLR) in Oberpfaffenhofen, Germany, where she is working as a research assistant and participates in the development of the Railway Collision Avoidance System (RCAS). She is working towards her Ph.D. at the University of Ulm focusing her research on performance analysis of MAC layers for safety applications in mobile ad-hoc multibroadcast networks and the development of robust MAC layers.

Thomas Strang is working as a senior researcher in the Institute of Communications and Navigation at the DLR in Oberpfaffenhofen. He joined DLR in 2000 where he is responsible for the Institute's program in transportation research since 2004, which includes new services for ITS and adhoc vehicle-to-vehicle communications. Since 2004 he has also been a professor for computer science at the University of Innsbruck. 